\title{
Diagnóstico ocupacional de las condiciones de trabajo en unas poblaciones agrícolas expuestas a plaguicidas organofosforados
}

Diagnosis of occupational working conditions in the agricultural stocks exposed to insecticides organophosphate

Diagnóstico de profissionais de trabalho nos estoques agrícolas expostos a inseticidas organofosforados

\section{Karen López ${ }^{1}$, David Alexander Villamil' ${ }^{2}$, Maira Fernanda Zambrano ${ }^{3} \llbracket C v L A C$}

Fecha correspondencia:

Recibido: abril 28 de 2015.

Revisado: junio 20 de 2015.

Aceptado: marzo 09 de 2016.

Forma de citar:

López K, Villamil D, Zambrano M. Diagnóstico ocupacional de las condiciones de trabajo en unas poblaciones agrícolas expuestas a plaguicidas organofosforados. Rev CES Salud Pública. 2016; 7(1): 17-24.

\section{Open access}

(c) Derecho de autor Licencia creative commons Ética de publicaciones Revisión por pares Gestión por Open Journal System ISSN 2145-9932

Sobre los autores:

1. Docente Investigadora Universidad Militar Nueva Granada, Facultad de Relaciones Internacionales, Estrategia y Seguridad.

Comparte

\section{Resumen}

Las actividades agrícolas constituyen gran parte de la actividad económica en Colombia, siendo esta una de las principales fuentes de empleo en los diferentes municipios del país, pero también una de las áreas productivas más vulnerables frente a seguridad y salud en el trabajo. Este proyecto tiene como objetivo identificar las condiciones de trabajo en las que se encuentran las poblaciones agrícolas de Natagaima y Puente Nacional asociando la percepción de los agricultores frente a condiciones de higiene y conductas seguras asumidas durante el uso de plaguicidas, analizadas en términos de porcentajes de las respuestas obtenidas por los agricultores a través encuestas.

Palabras clave: Uso de plaguicidas, exposición a plaguicidas, insecticidas organofosforados, ooblación rural, granjeros

\section{Abstract}

Agricultural activities constitute much of the economic activity in Colombia, which is one of the main sources of employment in the different municipalities of the country, but also one of the most vulnerable to safety and health at work productive areas. This project aims to identify the working conditions which are agricultural populations Natagaima and National Bridge associating the perception of farmers against hygiene and safe behavior adopted during the use of pesticides analyzed in terms of percentages the responses obtained by farmers through surveys.

Keywords: Pesticide utilization, pesticide exposure, insecticides organophosphate, rural population, farmers.

\section{Resumo}

Actividades agrícolas constituem a maior parte da atividade econômica na Colômbia, que é uma das principais fontes de emprego nos diferentes municípios do país, mas também um dos mais vulneráveis à segurança e 
2. Estudiante PegradoUniversidad Militar Nueva Granada, Facultad de Relaciones Internacionales, Estrategia y Seguridad.

3. Docente Investigadora Universidad Militar Nueva Granada, Facultad de Relaciones Internacionales, Estrategia y Seguridad. saúde no trabalho áreas produtivas. Este projeto tem como objetivo identificar as condições de trabalho que são populações agrícolas Natagaima e National Ponte associar a percepção dos agricultores contra higiene e comportamento seguro adotada durante o uso de pesticidas analisados em termos de percentagens as respostas obtidas pelos agricultores através de inquéritos.

Palavras-chave: Uso de praguicidas, exposição a praguicidas, inseticidas organofosforados, população rural, fazendeiros.

\section{Introducción}

En Colombia, las actividades de agricultura constituyeron el 9.3\% del PIB del país en el año 2012, en donde el departamento de Santander tuvo una participación del 7.5\% y el departamento del Tolima conto con un porcentaje de participación del 2.2\% (1). La población cultivadora de arroz estudiada pertenece a la zona arrocera centro de Colombia, caracterizada por una alta proporción de individuos mayores de 40 años.

El uso de plaguicidas organofosforados se ha convertido en una práctica común y necesaria para el sustento de los cultivos agrícolas a lo largo y ancho del país (2), sin embargo el conocimiento y aplicación de medidas de seguridad para estas sustancias agroquímicas es deficiente, esto genera un aumento silencioso de enfermedades generadas por estos productos que a pesar de estar regulados por la Dirección de Impuestos y Aduanas Nacionales (DIAN), siguen generando intoxicaciones (므, $\underline{4})$ debido la falta de información disponible, sumado a las escasas medidas de control por parte de los entes administrativos de los municipios y de la misma población agrícola.

En las poblaciones de Natagaima y Puente Nacional, se ha observado que las personas encargadas de la manipulación, no tienen una formación adecuada para realizar estas actividades, que por la naturaleza toxica de estos productos, deben ser manipulados bajo ciertos estándares como lo dicta el Decreto 1843 de 1991(므), en donde se reglamenta algunos capítulos de la ley 09 de 1979, sobre el uso de plaguicidas; los buenos hábitos ocupacionales y el correcto uso de elementos de protección individual, constituyen la primera línea de defensa contra la contaminación por plaguicidas organofosforados. Sin embargo la evidencia obtenida a través de encuestas aplicadas en las poblaciones objetivo, muestran, que no son tomadas en cuenta medidas de seguridad durante el uso de los plaguicidas, debido a diversos factores como el desconocimiento, costo, condiciones ambientales (temperaturas) o porque no son claros los beneficios.

\section{Materiales y métodos}

La selección de las poblaciones fue intencional o por conveniencia (ㅁ) después de establecer contacto con la alcaldía y los agricultores de los municipios Natagaima y Puente Nacional. Las bases de datos de los trabajadores del sector agrícola en los municipios fueron solicitados a las Alcaldías Municipales, de las cuales se estimó la población expuesta. La población agricultora informal encuestada corresponde a un grupo de 48 hombres y mujeres entre los 21 y 78 años cuya principal actividad es el cultivo de arroz en el municipio de Natagaima. En el municipio de Puente Nacional, Santander, la población encuestada fue de 51 agricultores informales en un rango etario de 17 y 78 años dedicados a la producción agrícola representada en su mayoría por el cultivo de caña panelera y piña (). 
Se realizó la aplicación de una encuesta para indagar acerca del estado y antecedentes de salud (으), hábitos y comportamientos relacionados con la actividad laboral de los participantes (2-11) y participación previa en programas nacionales de vigilancia y control de exposiciones a plaguicidas.

Se analizaron los resultados en términos de porcentajes de las respuestas a cada pregunta de la encuesta aplicada a los participantes.

El presente estudio fue de participación voluntaria, en el que a través de consentimiento informado por escrito, les fue advertido a los participantes el nivel de riesgo de la investigación, según lo establecido por la resolución 008430 de 1993 del Ministerio de Salud de Colombia. Adicionalmente la privacidad de los participantes y los datos fue protegida utilizando un sistema de codificación.

\section{Resultados}

Los agricultores de Natagaima, son (9\%), más propensos a adquirir una intoxicación por vía gastrointestinal que los trabajadores de Puente Nacional teniendo en cuenta que manifiestan consumir bebidas hidratantes durante la aplicación de plaguicidas. (Figura 1)

El $86 \%$ de la población de Puente Nacional, usa más de una vez las mismas prendas, con las que aplican los plaguicidas, refiriendo olvido o poca relevancia frente a este hábito de higiene. (Figura 2)
Figura 1. Proporción de agricultores que consumen alimentos o bebidas durante el manejo de plaguicidas

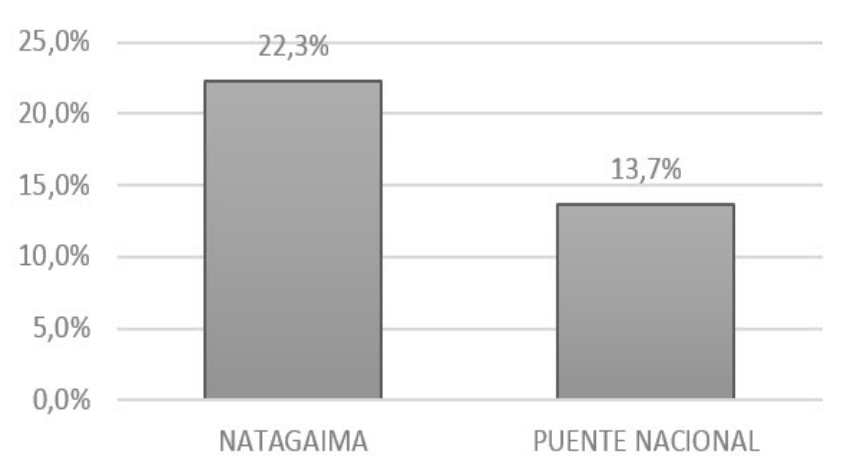

Tomar un baño después de cada jornada, es considerado necesario por parte de la mayoría de agricultores encuestados en las dos poblaciones. (Figura 3)

De los elementos de protección individual el respirador se constituye como el de menor utilización, registrando un $0 \%$ de uso en la población de Natagaima y sólo el $5,9 \%$ en la población de Puente Nacional. (Figura 4)

El factor incomodidad se constituye como una de las razones más frecuentes para no usar los elementos de protección personal (EPP), seguida por la dificultad de adquirirlos por costos elevados, el desconocimiento acerca de los elementos a utilizar y la utilidad que prestan, también se encuentran establecidas dentro de las variables que impiden el uso de estos. (Figura 5) 
En promedio el $73,4 \%$ de la población agricultora, realiza disposición final de residuos de plaguicidas inadecuada, sólo el 26, 6 \% en promedio conocen el lugar dispuesto por los municipios para este fin. (Figura 6 )

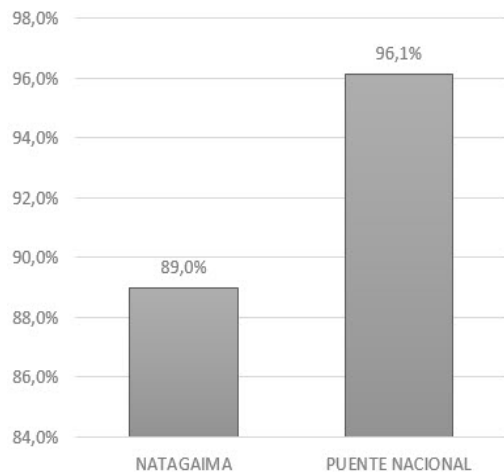

Figura 3. Proporción de agricultores que se bañó después de la jornada de fumigación

Figura 4. Uso de elementos de protección personal por los agricultores en las poblaciones de Natagaima y Puente Nacional
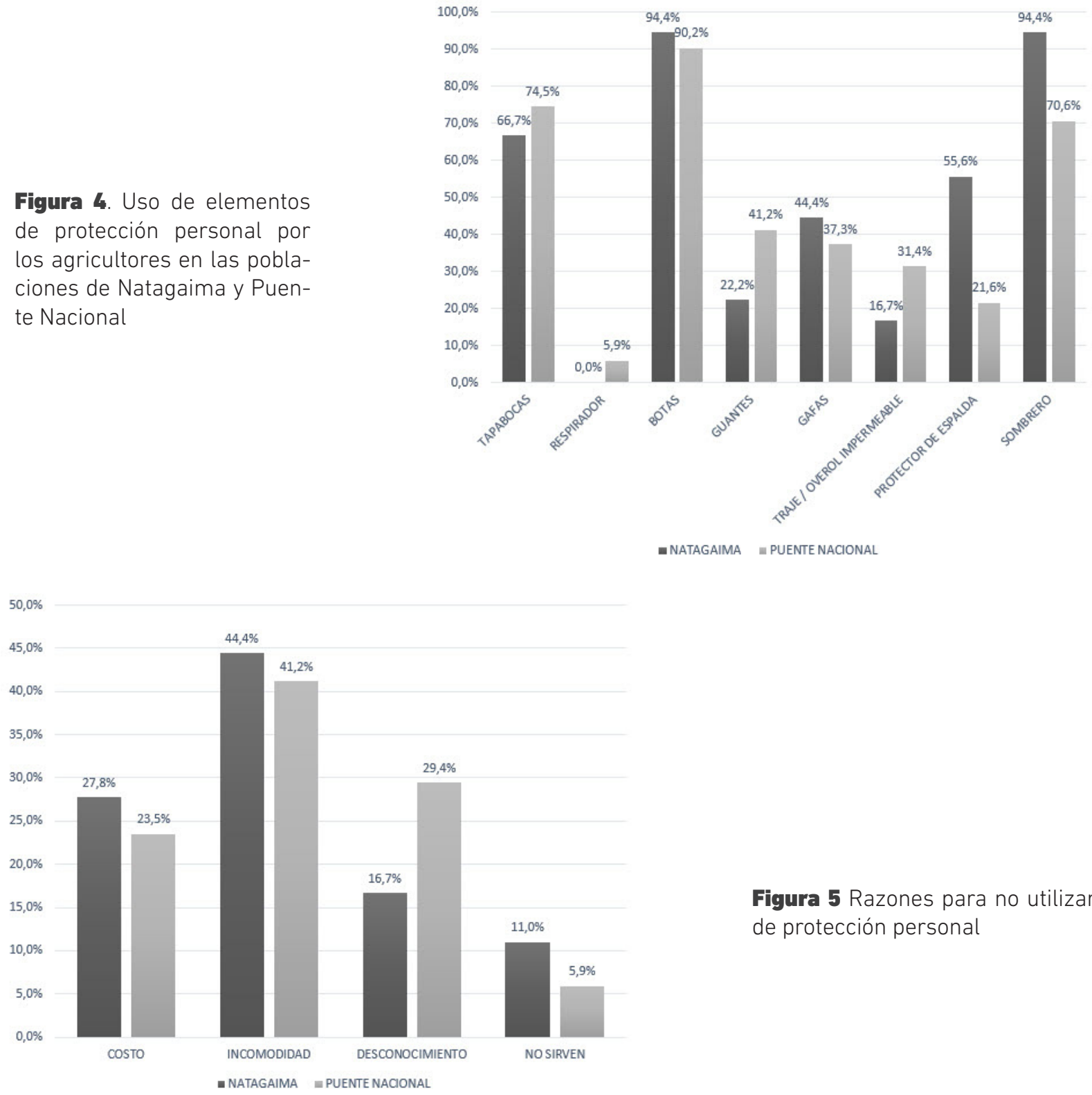

Figura 5 Razones para no utilizar los elementos de protección personal 


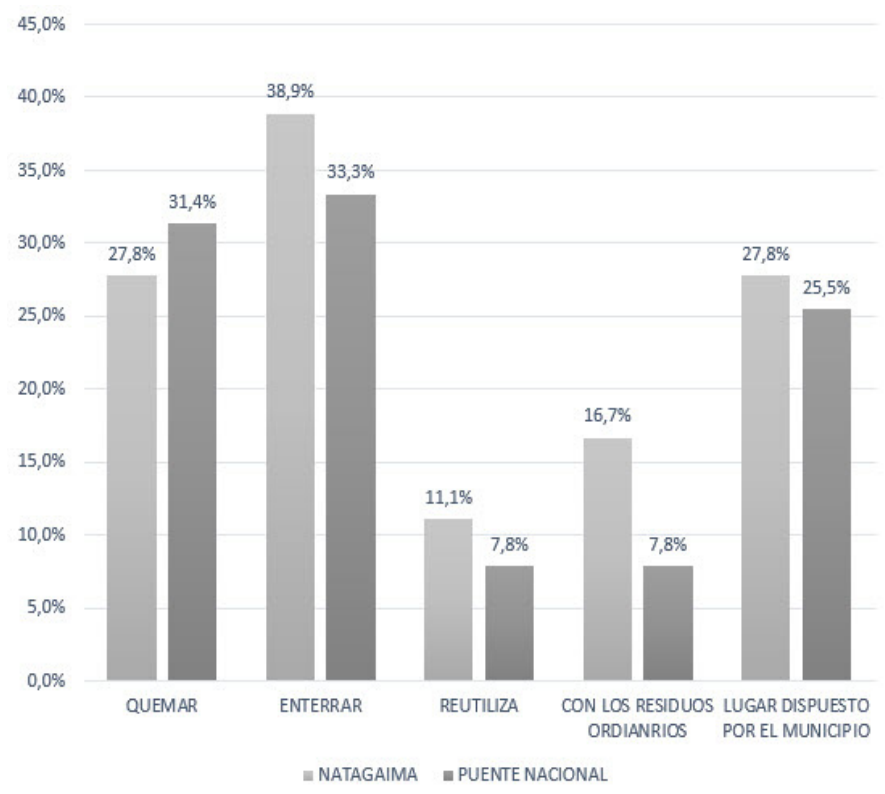

Figura 6. Tratamiento de los envases de los plaguicidas

\section{Discusión}

Los cultivos típicos de las dos poblaciones son objetivo de numerosas plagas, propiciando así el uso de grandes cantidades de plaguicidas para controlarlas; si se tiene en cuenta que entre el año 2012 y 2013, se ha cultivado un aproximado de 19867.7 hectáreas (12), la exposición a plaguicidas ha sido considerable, lo cual resulta preocupante teniendo en cuenta el bajo nivel de capacitación en la manipulación de plaguicidas, dado que ninguno de los agricultores vinculados al estudio cuenta con certificación para ejecutar actividades asociadas al manejo de los mismos. Dicha certificación es exigida por la normatividad colombiana la cual establece que toda persona que aplique plaguicidas debe estar registrada y haber recibido capacitación teórico-práctica por parte de un ente educativo autorizado (4). Teniendo en cuenta que la mayoría de los cultivadores de arroz del Tolima son pequeños agricultores arrendatarios de las tierras que cultivadas (13), es poco frecuente que reciban capacitaciones pues no se encuentran vinculados a los sistemas de gestión de seguridad y salud en el trabajo en asociación a la informalidad de su labor.

Las elevadas temperaturas alcanzadas en la región del Tolima y Santander, generan alta sudoración, fatiga y disconfort térmico en los agricultores durante la aplicación de los plaguicidas lo cual se asocia a la incomodidad referida como una de las causas que impactan el porcentaje de uso de elementos de protección individual (EPI) en la población.

La dotación completa para el manejo adecuado de plaguicidas en promedio requiere una inversión aproximada de U\$45 (COL\$85.000), un agricultor promedio devenga menos de un SMLV, que debe invertir en alimentación, educación y servicios públicos para él y su familia, situación relacionada con la percepción de costos elevados que impactan la utilización de los EPI, hallazgo reportado por Feola y Binder en el 2010b y MacFarlane en 2013. 
Se sabe que hay una alta influencia de las normas sociales en las comunidades de agricultores, que estarían influyendo en la deficiencia de uso de elementos de protección personal $(\underline{14}, \underline{15})$ como por ejemplo la percepción generalizada en la comunidad de que los elementos de protección personal son poco importantes en el desarrollo de la actividad agrícola (16), percepción evidenciada en las poblaciones de Natagaima y Puente Nacional que muestra desconocimiento sobre los posibles efectos adversos para la salud generados por los plaguicidas, en asociación a la poca divulgación de las normas existentes frente a dicha temática, lo que predispone a las poblaciones a desarrollar toxicidad por exposición aguda o crónica a plaguicidas durante sus labores agrícolas, en asociación a la falta de presencia del Estado en el control e inclusión de los agricultores informales en programas preventivos de seguridad y salud en el trabajo además de capacitación en buenas prácticas agrícolas. Adicionalmente inexistente medición de colinesterasa eritrocitaria referenciada por los agricultores, demuestra falta de implementación de los programas de salud pública para el control de intoxicaciones por plaguicidas, generando incertidumbre acerca de la disponibilidad de recursos para la realización de las mismas o para el acceso de los agricultores a los laboratorios de referencia.

A este contexto se le suma como agravante, la escasa información en Colombia relacionada con la frecuencia de intoxicaciones y sus causas dentro la población agricultora además de la falta de control por parte del Estado colombiano sobre temas como, la regulación de sustancias agroquímicas, capacitación a poblaciones expuestas a plaguicidas y la disposición final de los envases que contienen los plaguicidas, siendo esta última una de las causas generadoras de contaminación ambiental paralela al posible daño para la salud en los pobladores de la comunidad en general.

En cuanto a lo relacionado a la falta de control de los agroquímicos es importante relacionar que los distribuidores de plaguicidas como parte de la cadena de abastecimiento, tienen una responsabilidad, pues estos deben brindar suficiente información de los plaguicidas que comercializan, con el fin de facilitar la prevención de efectos adversos en la salud de las personas y animales así como también daños en el ambiente.

Dado que la población participante nunca ha sido objeto de programas de biomonitoreo, se hace necesaria la modificación de las estrategias nacionales de prevención de intoxicación por exposición ocupacional a plaguicidas, de carácter incluyente para los pequeños agricultores en programas integrales de salud pública que contemplen capacitación, seguimiento, vigilancia y control en la aplicación segura de plaguicidas y especialmente en los Sistemas de Seguridad y Salud en el Trabajo no sólo aplicables para las empresas públicas y privadas sino también para los municipios cuya actividad económica principal relacione la agricultura que en su mayoría es ejecutada por campesinos que constituyen su actividad laboral como informal.

\section{Agradecimientos}

El presente trabajo de investigación fue financiado por la Universidad Militar Nueva Granada, los autores expresamos nuestro agradecimiento a la Vicerrectoría de Investigaciones, a la Facultad de Relaciones Internacionales, Estrategia y Seguridad, al Programa Administración de la Seguridad y Salud Ocupacional, quienes mediante el semillero de investigación en toxicología ocupacional apoyaron el buen desarrollo del mismo. Así mismo se agradece la participación de los estudiantes que han hecho parte de toda la labor investigativa para poder presentar estos resultados que se espera aporten al avance de la seguridad y salud en el trabajo del País. 


\section{Bibliografía}

1. Montoya JD. Actividades económicas de Colombia, 2012, 10 de Marzo [citado Marzo 03.] en: Actividades económicas. [http://www.actividadeseconomicas. org/]. Recuperado de http://www.actividadeseconomicas.org/2012/03/principales-actividades-economicas-de.html\#.VBr4x I5MnV

2. Instituto Colombiana Agropecuario (ICA). Estadísticas de comercialización de plaguicidas químicos de uso agrícola 2010. Bogotá: ICA; 2011. http://www.ica. gov.co/Areas/Agricola/Servicios/Regulacion-y-Control-de-Plaguicidas-Quimicos/Estadisticas-(1)/2010/Comercializacion-plaguicidas-2010(int)-(4).aspx

3. Instituto Nacional de Salud. Protocolo de Vigilancia en Salud Pública 2014. Bogotá: SIVIGILA; 2014. Serie de protocolos de vigilancia: PRO-R02.006 http://www. ins.gov.co/lineas-de-accion/Subdireccion-Vigilancia/sivigila/Protocolos\%20SIVIGILA/PRO\%20ESAVI.pdf

4. Instituto Nacional de Salud. Informe Quincenal Epidemiológico Nacional. Caracterización epidemiológica de las intoxicaciones por plaguicidas en Colombia 2011-2012, Volumen 18 numero 20. Bogotá: IQEN; 2013. Serie de informes epidemiológicos. http://www.ins.gov.co:81/iqen/IQUEN/IQEN\%20vol\%2018\%20 2013\%20num\%2021.pdf

5. "Por el cual se reglamentan parcialmente los títulos III, V, VI, VII y XI de la Ley 09 de 1979, sobre uso y manejo de plaguicidas". Decreto 1843 de 1991., (22 de Julio de 1991). http://www.andi.com.co/es/PC/SobProANDI/Documentos\%20 Sobre\%20Procultivos\%20ANDI/Decreto1843 1991 plaguicidas.pdf

6. Jiménez ME. Martínez V: Efecto del tiempo y las condiciones de almacenamiento sobre la actividad de las colinesterasas / Effect of time and the conditions of storage in the activity of the cholinesterases. Revista médica de Costa Rica. Oct-Dic de 2000; disponible en http://bases.bireme.br/cgibin/wxislind.exe/iah/ online $/$ ? IsisScript=iah/iah. $x$ is \&src=google\&base $=$ LILACS\&lang=p\&nextAction $=1$ $\underline{n k \& \text { exprSearch }=279842 \& \text { indexSearch }=I D}$

7. Agronet [http://www.agronet.gov.co/agronetweb1/]. Bogotá: Producción en el departamento de Santander: Reporte de producción agrícola por departamento. Disponible en : http://www.agronet.gov.co/www/htm3b/excepcionesNuke/cargaNet/netcarga138.aspx?cod $=138 \&$ Depto $=68 \&$ submit $=$ Ver $\% 20$ Reporte\&reporte=Producci\%F3n\%20agr\%EDcola \% 20 por $\% 20$ departamento\&file $=2006102316652$ ReportEVAPorDepartamento.rpt\&codigo $=$ $138 \&$ excepcion $=1 \&$ fechal $=2012 \&$ fechaF $=2013$

8. "Por el cual se Reglamenta Parcialmente el Título IX de la Ley 09 de 1979, en cuanto a Funcionamiento de Establecimientos Dedicados a la Extracción, Procesamiento, Conservación y Transporte de Sangre Total o de sus Hemoderivados, se Crean la Red Nacional de Bancos de Sangre y el Consejo Nacional de Bancos de Sangre y se dictan otras disposiciones sobre la materia". Decreto 1571 de 1993, "de los donantes" Capitulo III, (12 Agosto de 1993). https://www.minsalud. gov.co/Normatividad_Nuevo/DECRETO\%20\%201571\%20DE\%201993.pdf 
9. Icontec internacional. guía para la identificación de los peligros y la valoración de los riesgos en seguridad y salud ocupacional (GTC45). Bogotá: ICONTEC; 2010. http://idrd.gov.co/sitio/idrd/sites/default/files/imagenes/gtc450.pdf

10. "Por el cual se reglamentan parcialmente los títulos III, V, VI, VII y XI de la Ley 09 de 1979, sobre uso y manejo de plaguicidas". Decreto 1843 de 1991., (22 de Julio de 1991). http://www.andi.com.co/es/PC/SobProANDI/Documentos\%20 Sobre\%20Procultivos\%20ANDI/Decreto1843 1991 plaguicidas.pdf

11. Barnett M, Calvert GM. 2005. Pesticide-related illness and injury surveillance: a how to guide for state-based programs. Publication number 2006-102. Department of Health and Human Services, Centers for Disease Control and Prevention, National Institute for Occupational Safety and Health; 2005. http://www. cdc.gov/niosh/docs/2006-102/pdfs/2006-102.pdf

12. Agronet [http://www.agronet.gov.co/agronetweb1/]. Bogotá: Producción en el departamento de Santander: Reporte de producción agrícola por departamento. Disponible en : http://www.agronet.gov.co/www/htm3b/excepcionesNuke/cargaNet/netcarga138.aspx?cod $=138 \&$ Depto $=68 \&$ submit $=$ Ver $\% 20$ Reporte\&reporte=Producci\%F3n\%20agr\%EDcola\%20por\% 20 departamento\&file $=2006102316652$ ReportEVAPorDepartamento.rpt\&codigo $=$ $138 \&$ excepcion $=1 \&$ fechal $=2012 \&$ fechaF $=2013$

13. Federación Nacional de Arroceros. [http://www.fedearroz.com.co/new/index.php]. Bogotá: Tercer censo nacional arrocero, Zona Centro. 2008. Disponible en: http:// www.fedearroz.com.co/doc economia/Censo\%20111\%20\%20Centro\%2012\%20 marzo\%202008.pdf

14. Feola G, Binder C. Identifying and investigating pesticide application types to promote a more sustainable pesticide use. The case of smallholders in Boyacá, Colombia. Crop Protection. 2010. 29. 612-622. https://core.ac.uk/download/ pdf/107922.pdf

15. Feola G, Binder C. Why don't pesticide applicators protect themselves? Exploring the use of personal protective equipment among Colombian smallholders. International Journal of Occupational and Environmental Health. 2010. 16 (1). 11-23. http://www.ncbi.nlm.nih.gov/pubmed/20166315

16. Gomes J, Lloid OL, Revitt DM. The influence of personal protection, environmental hygiene and exposure to pesticides on the health of inmigrant farm workers in a desert country. International Archives of Occupational and Environmental Health. 1999.72. 40-45. http://www.ncbi.nlm.nih.gov/pubmed/10029229 